\title{
Network Maintenance Optimal Strategy Model Building based on Game Analysis and Hierarchical Clustering
}

\author{
Pengfei Xu \\ Southwest Petroleum University, Chengdu, Sichuan, China 610500, China.
}

\begin{abstract}
This paper proposes the novel network maintenance optimal strategy model building based on game analysis and hierarchical clustering. The urban water supply system is city people life and economic development important infrastructure, and is lost the distribution network of water supply system comprised of the urban water supply water source and feed-water treatment system. Economic development has not reduced the demand for water and people's living standards improve the quality of water requirements are also rise and this to the urban water supply pipe network construction has brought a certain amount of the pressure to ensure adequate water supply but also to ensure the quality of water supply. We propose the new idea on the optimal strategy model to propose the reasonable countermeasures. The effectiveness is proved in the paper.
\end{abstract}

Keywords: Network Maintenance; Game Analysis; Hierarchical Clustering; Strategy.

\section{Introduction}

The urban water supply system is city people life and economic development important infrastructure, and is lost the distribution network of water supply system comprised of the urban water supply water source and feed-water treatment system. For 20 years, along with the rapid growth of economy, speeding up of urbanized degree, the scope of large cities expanded unceasingly, we need to change to expand with according to the urban development many networks newly-built that has replaced the massive aged pipelines, but still had for the number extremely numerous the pipeline that should retire was operating. In the system of water resources shortage and serious water pollution, water cost is increasing, and the contradiction between supply and demand of urban water expands unceasingly, depth and systematic study of the problem of the leakage, and actively explore the effective loss of control methods for the effective use of water resources that reduce environmental hazards to reduce operating and the management costs that will improve service quality, water supply enterprises to improve economic and social benefits with special significance.

At present, the leakage detection equipment commonly used are missed bar; electronic leakage listening detector; noise automatic recorder; related instrument etc. The water supply enterprise shall be determined according to the geographical position, economic and technical conditions. Based on the literature review, the current methodologies can be summarized as the follows.

(1) Passive leak detection law.

Passive leak detection method mainly relies on the specialized personnel to carry out the inspections and leaks and users reported two ways. Passive leak detection method of investment, with a wide range of the social and mass.

(2) Sound leak detection method.

The sound listens to the leak detection law to carry on the examination leaking point according to the above audio frequency, the sound listens to the leak detection law to be divided into valve pin listening and ground listens two types, the former is used to search the water leakage the clue and scope, the abbreviation leaking point pre-localization, while the latter is used to determine that leaks the water drop position, the abbreviation leaking point pinpointing.

\section{(3) The region installs the table law.}

If we put the area of the user meter and regional meter reading date on the same day, and the meter reading time difference to a very small extent, if the accuracy of the water meter to meet the requirements, the difference between the two is the amount of water lost during the meter reading interval. 
In this paper, to deal with the traditional system's drawbacks, we conduct research on the network maintenance optimal strategy model building based on game analysis and hierarchical clustering. In the later section, we will discuss the method with background analysis.

\section{The Proposed Method}

\subsection{The Hierarchical Clustering.}

The sequence cluster besides must have the reasonable similar measure method, but must select the reasonable clustering method, the hierarchically clustering in the sequence cluster is one of the most commonly as used cluster arithrnetics while the condensation hierarchically clustering law is also most preferred. This article proposed a new sequence similar measure method. This method first bases on within two sequences to have the match mark number computation initial similarity of statistical significance, subsequently uses the length standard factor to reduce effect of the sequence length on the similarity. Based on this metric, a new clustering hierarchical clustering method based on single link is proposed. This method constructs loop-free graphs for symbol sequences from bottom to top. By introducing the definition of link degree between samples, the link degree of link samples is calculated of the class.
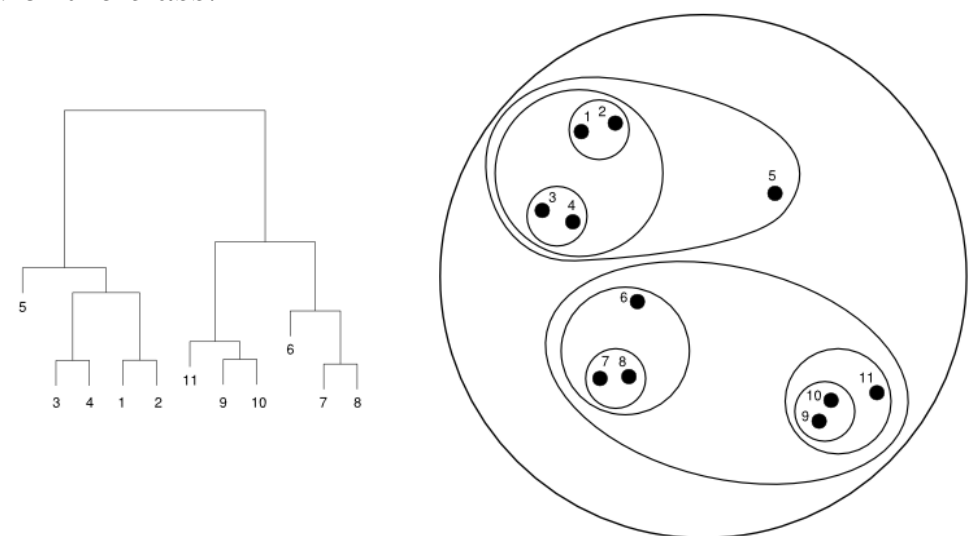

Fig. 1 Hierarchical Clustering Principle Demonstration

As demonstrated in the figure one, the principles are demonstrated. A new clustering algorithm in the segmentation graph, the inter cluster similarity between samples converted to link degree, the link degree of integrated cluster distance and inter cluster link samples and two factors of the nearest neighbor distance to avoid the traditional agglomerative algorithm that only according to the distance between cluster classification problems. Through the cellular automaton construction hierarchically clustering evolution model, can realize the merges of several varieties in the same evolved cycle has manifested the characteristics of parallel computing. If there is null cycle not to have merge then the length of stride of null cycle becomes bunch and distance measuring between varieties that can be the number of clustering definite basis. The formula one defines the standard.

$$
k=\frac{\sum_{i=1}^{n}\left[k-\operatorname{distance}\left(P_{i}\right)\right]}{N}
$$

In the stratification structure has two joint connection ways: a) variety internal node connects, can in the smallest subtree through the Prim algorithm connection production variety; b) among varieties the joint connection, among the varieties connects realizes through the connection variety first node, and therefore needs to define the variety first selection criteria. Under this basis, we can summarize the proposed clustering algorithm as the follows.

- Extracting feature: According to the prior knowledge of domain experts and combining with the specific purpose of data, the relevant feature is selected from the original dataset, and some irrelevant feature vectors are discarded to reduce the non-correlation data clustering results.

- Cluster: After suitable pretreatment data that obtains as input, considered that the data the structure and type, determined the distance measuring model of matching that will choose the suitable cluster arithrnetic to carry on the cluster. 
- Evaluating the clustering results: If we use or trust the clustering results without thinking, we will make our decisions blind or biased. Therefore, the evaluation of clustering effect is one of the most important steps in cluster analysis. Evaluation usually requires domain experts, and even multi-stakeholder participation, common analysis and interpretation of the data revealed by cluster characteristics and the inherent law and the real world consistency.

\subsection{The Game Analysis.}

Oligopoly refers to only the few companies control the commodity market structure. In the economics, several game models have been proposed to simulate the oligopolistic behavior in the market. Cournot game model is one of the most commonly used. It is an oligopolistic model of production with the assumption that the oligarchs have complete information about their own and their competitors' production decisions and profits, and that they are perfectly rational, so that the oligarchs can achieve equilibrium only once in the game.

The realistic oligopoly market is very complex, and the enterprise grasps the market information quantity to be not necessarily coordinated, and enterprise policy-maker's ability to the information processing mutually is not same. This article considers in the market information asymmetrical situation, information content that because rational level different individuals grasp, and is different to the reaction capacity of market uses completely different competitions to produce the strategy: A side uses the time delay limited rationality to compete to produce the decision-making, but another side uses the optimal response. According to the correlation theories of power system has carried on the qualitative analysis to the configuration model evolution, discussed the model Nash point of the equilibrium existence and stability shown as the figure 2 .

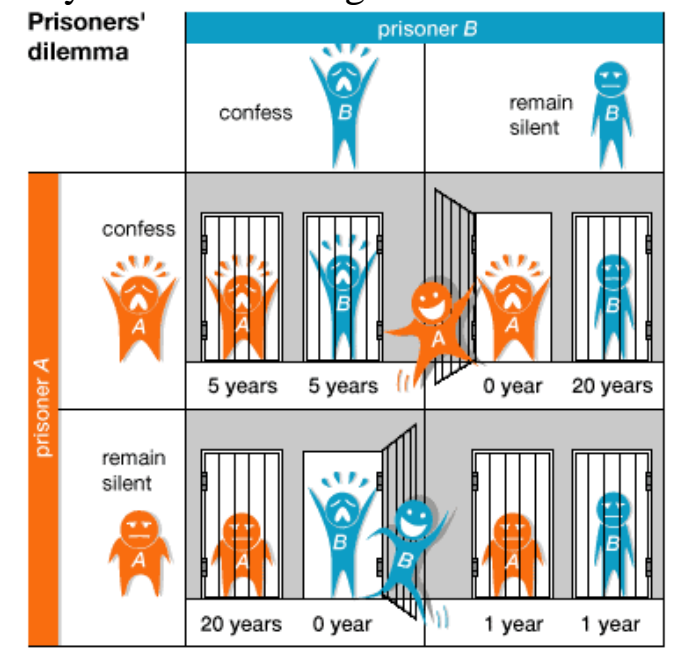

Fig. 2 The Visualized Demonstration of the Game Analysis

We can see according to the above analysis, regarding the double oligarch competition of different rational and different cost structure, after multiple gambling will have complex situations. Bounded rational game and the adjustment of the marginal profit as the change of the reaction speed and the adaptation speed to the game process can change the stability of the game equilibrium, so that the market enters the chaotic state. For lower response and adjustment speed, the game will tend to only Nash equilibrium, but with the increase of reaction and adaptation speed, Nash equilibrium will lose its stability, branch and chaos will appear.

The real world in a large number of competitions due to market disorder caused by the phenomenon of a more reasonable explanation, as the model can be shown as follows.

$$
\begin{aligned}
& q_{1}^{\prime}=\operatorname{argmax} \prod_{l}\left(q_{1}, q_{2}^{e}(t+1)\right) \\
& q_{2}^{\prime}=\operatorname{argmax} \prod_{2}\left(q_{1}^{e}(t+1), q_{2}\right)
\end{aligned}
$$

\subsection{Network Maintenance Optimal Strategy Model.}

Water supply pipe network has good sealing performance, good water quality good hydraulic conditions equipment control spirit and so on. The water supply from the water plant to the user, to go 
through a long pipeline, the pipe network can actually be seen as a relatively large reactor, the wall is smooth, non-scaling and pipeline flow, the factory water is not complete chemical reaction is in the pipe network to continue, the total length of the pipe network of the water supply up to thousands of kilometers, hundreds of kilometers less, and some chlorine water and pipe wall new contacts, there may be a new reaction as these reaction is sensory and recognized as the biological or chemical. Based on the mentioned theories, here, we give the suggestions on the maintenance as the follows.

\subsubsection{Strengthen the importance of the network maintenance.}

Strengthen the construction supervision, the implementation of the construction monitoring daily, weekly system. The construction supervisor immediately reported to investigators on-site pipeline inspection operations, the detection staff to confirm the pipeline, the guard will be on-site all the water supply line marked with a red paint from the site in a prominent position, the construction section after the guardians inventory of water supply assets.

\subsubsection{Application and spreading new tubing and speeds up the obsolete pipe network to transform the step.}

In order to ensure compliance with the relevant requirements of the national water quality standards for fittings, pipes and equipment into the water supply network, it is needed to strengthen the real estate construction of the indoor water supply pipeline for quality inspection and receiving management.

\subsubsection{Strictly strengthen the maintenance of large diameter pipe network valves.}

We must dock to the sewer pipe or access river bed the maintenance management of excretion water valve strengthens making it maintain complete condition. In addition, but should also enhance this length of pipe the effort of inspecting the maintenance in the flood season, achieves discovers pipe-line valve breakdown prompt repair processing early.

\section{Summary}

This paper proposes the novel network maintenance optimal strategy model building based on game analysis and hierarchical clustering. Economic development has not reduced the demand for water and people's living standards improve the quality of water requirements are also rising and this to the urban water supply pipe network construction has brought a certain amount of the pressure to ensure adequate water supply but also to ensure the quality of water supply. Therefore, the maintenance of water supply pipe network technology has also become an era of demand. This paper integrates the game analysis and hierarchical clustering to propose the novel network maintenance optimal strategy model, in the future we will apply this to test the general performance.

\section{References}

[1] Sarkkola, S., et al. "Domination of growing-season evapotranspiration over runoff makes ditch network maintenance in mature peatland forests questionable." Mires and Peat 11.2 (2013): 1-11.

[2] Goel, Asvin, and Frank Meisel. "Workforce routing and scheduling for electricity network maintenance with downtime minimization." European Journal of Operational Research 231.1 (2013).

[3] Manshaei, Mohammad Hossein, et al. "Game theory meets network security and privacy." ACM Computing Surveys (CSUR) 45.3 (2013): 25.

[4] Bi, Chujian, et al. "SAR image restoration and change detection based on game theory." Intelligent Computing and Internet of Things (ICIT), 2014 International Conference on. IEEE, 2015.

[5] Suzuki, Ryota, and Hidetoshi Shimodaira. "Hierarchical clustering with P-values via multiscale bootstrap resampling." R package (2013).

[6] Langfelder, Peter, and Steve Horvath. "Fast R functions for robust correlations and hierarchical clustering." Journal of statistical software 46.11 (2012). 